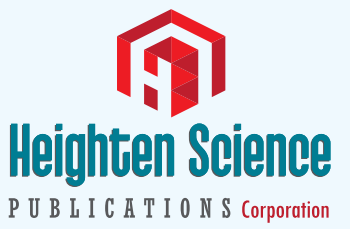

ISSN

2573-7724
*Address for Correspondence: Yuanshui Zheng, Atlantic Health System, Oklahoma State University, USA, Tel: 405-248-8929 (C); Email: yuanshuizheng@yahoo.com

Submitted: 09 August 2018

Approved: 12 September 2018

Published: 13 September 2018

Copyright: @ 2018 Zheng Y, et al. This is an open access article distributed under the Creative Commons Attribution License, which permits unrestricted use, distribution, and reproduction in any medium, provided the original work is properly cited.

Keywords: Uniform scanning; Proton therapy; Lung cancer; Adaptive radiotherapy

Check for updates

\title{
Adaptive planning and toxicities of uniform scanning proton therapy for lung cancer patients
}

\author{
Yuanshui Zheng ${ }^{1 *}$, Hardev Singh ${ }^{2}$, Suresh Rana ${ }^{3}$, Gary Larson ${ }^{2}$, \\ Prabhu Kiran ${ }^{2}$, Lucius Doh ${ }^{2}$ and James Wong ${ }^{1}$ \\ ${ }^{1}$ Atlantic Health System, 100 Madison Avenue, Morristown, NJ 07960, USA \\ 2ProCure Proton Therapy Center, 5901 W Memorial Rd, Oklahoma City, OK 73142, USA \\ ${ }^{3}$ Miami Cancer Institute, Proton Therapy, 8900 North Kendall Drive, Miami, FL 33176, USA
}

\section{Abstract}

Purpose: Adaptive planning is often needed in lung cancer proton therapy to account for geometrical variations, such as tumor shrinkage and other anatomical changes. The purpose of this study is to present our findings in adaptive radiotherapy for lung cancer using uniform scanning proton beams, including clinical workflow, adaptation strategies and considerations, and toxicities.

Methods: We analyzed 165 lung patients treated using uniform scanning proton beams at our center. Quality assurance (QA) plans were generated after repeated computerized tomography (CT) scan to evaluate anatomic and dosimetric change during the course of treatment. Plan adaptation was determined mutually by physicists and physicians after QA plan evaluation, based on several clinical and practical considerations including potential clinical benefit and associated cost in plan adaption. Detailed analysis was performed for all patients with a plan adaptation, including the type of anatomy change, at which fraction the adaption was made, and the strategy for adaptation. Toxicities were compared between patients with and without plan adaptation.

Results: In total, 32 adaptive plans were made for 31 patients out of 165 patients, with one patient undergoing adaptive planning twice. Anatomy changes leading to plan adaptation included tumor shrinkage (17), pleural effusion (3), patient weight loss (2), and tumor growth or other anatomy change (9). The plan adaptation occurred at the 15 th fraction on average and ranged from the $1 \mathrm{st}$ to 31 st fraction. Strategies of plan adaptation included range change only (18), re-planning with new patient-specific hardware (9), and others (5). Most toxicities were Grade 1 or 2, with dermatitis the highest toxicity rate.

Conclusions: Adaptive planning is necessary in proton therapy to account for anatomy change and its effect on proton penetration depth during the course of treatment. It is important to take practical considerations into account and fully understand the limitations of plan adaptation process and tools to make wise decision on adaptive planning. USPT is a safe treatment for lung cancer patients with no Grade 4 toxicity.

\section{Introduction}

Lung cancer remains to be the leading cause of cancer death in the United States with over 154,000 lung cancer deaths estimated in 2018 [1 https://www.cancer.org/ cancer/non-small-cell-lung-cancer/about/key-statistics.html]. Proton beam therapy (PBT) has been increasingly used for lung cancer, mainly because of its dose advantage over conventional radiation therapy such as 3D conformal radiation therapy (3DCRT) and intensity modulated radiation therapy (IMRT), and the increased number of proton therapy centers becoming available. Based on how a proton beam is spread out laterally and in depth, there are three main types of proton delivery techniques: passive scattering proton therapy (PSPT), uniform scanning proton therapy (USPT), and pencil beam scanning (PBS) [1-3]. 
Previous studies have shown that compared to 3D conformal or intensity modulated photon radiation, proton beams can better spare the lung, esophagus, heart, cord, and other normal tissues while delivering the same or higher dose to the treatment target, potentially leading to better tumor control and less toxicity [4-12]. Using PSPT, Chang et al. reported that the dose to normal tissues and the integral dose to patients with non-small cell lung cancer (NSCLC) were significantly reduced compared to 3DCRT and IMRT [11]. Kadoya et al., reported that PSPT significantly reduced lung dose compared to stereotactic body radiation therapy (SBRT) for Stage I non-small-cell lung cancer [10]. For SBRT with a prescribed dose for $66 \mathrm{~Gy}$, the mean dose, V5, V10, V15, and V20 were $7.8 \mathrm{~Gy}, 32 \%, 21.8 \%, 15.3 \%$, and $11.4 \%$ for SBRT, but reduced to $4.6 \mathrm{~Gy}$, $13.2 \%, 11.4 \%, 10.1 \%$, and $9.1 \%$ for proton therapy, respectively. In addition to less dose to the lung, Hoppe et al. reported that PSPT delivered less dose (D0.1cc and D5cc) to the heart, esophagus and bronchus compared to SBRT [9]. For locally advanced Stage III NSCLC patients, Wu et al. found that proton beam therapy was feasible and would spare lungs, heart and spinal cord better compared to 3DCRT[5]. Because of its less proximal dose and better optimization capability, IMPT can further reduce doses to normal tissues, such as the lung, spinal cord, heart, and esophagus, compared to PSPT and IMRT for extensive Stage IIIB NSCLC, as reported by Zhang et al. [13]. Stuschke et al., also reported that IMPT was advantageous in terms of lung sparing compared to both Tomotherapy and IMRT [7]. The dosimetry of USPT for lung cancer was similar to PSPT, as reported by Zheng [14].

While proton beams provide a superior dose distribution due to their finite ranges, the dose advantage can be compromised due to range uncertainties and changes from the daily setup variation and anatomical change. This is of particular concern for lung cancer therapy since progressive anatomical changes such as tumor shrinkage and positional changes are often observed (Toward Adaptive, $1^{\text {st }}$ paragraph). Hui et al., found that inter-fractional motion and anatomic change could lead to a result of up to $8 \%$ reduction of CTV coverage and a mean 4.4 CGE maximum dose increase of spinal cord [20]. Koey et al., reported that without adaptive planning, target coverage could be dropped to below $60 \%$ for some lung cancer case undergoing proton therapy [21]. To account for the dose disturbance due to the anatomical changes, adaptive planning is often required to ensure optimal target coverage and normal tissue sparing during the whole course of proton therapy.

Uniform scanning proton therapy (USPT) has been used at our proton center for lung cancer patient treatment. Its use in adaptive planning of lung cancer patients and its toxicities has been rarely reported. The purpose of this study is to illustrate our workflow, strategies, and practical considerations in adaptive planning of lung cancers using USPT. In addition, the acute and late toxicities for lung cancer patients treated at our center were also analyzed.

\section{Materials and Methods}

\section{Treatment techniques}

Simulation: Each patient was simulated using GE CT scanner (Lightspeed 16, Wisconsin) and an RPM system (Varian, Palt, CA) for motion monitoring. Patients typically lied supine, were immobilized with a vacuum bag, which was on top of an index fixed framing device (wing board), and had their arms up and hands holding the pegs on the wing board. The patient was scanned at $2.5 \mathrm{~mm}$ slice thickness. If contrast is used, one computerized tomography (CT) scan should be taken before the contrast is injected in addition to one after the injection. The CT data with intravenous contrast will be used primarily for target delineation, and the CT data set without contrast will be used for dose calculation. Tumor motion was evaluated by physics team. If the magnitude of motion was too big (e.g., larger than $1 \mathrm{~cm}$ ), either motion management would be applied, such as the use of compression belt, or conventional photon therapy would be used for treatment instead of proton therapy. 
Treatment planning: Based on the 4D CT scan, the internal gross target volume (IGTV) was contoured on the maximum intensity pixel (MIP) images and expanded 7-10 mm to generate the clinical target volume (CTV). The CTV was further expanded by $5 \mathrm{~mm}$ to obtain the planning target volume (PTV). Treatment planning and dose calculation were performed on the average CT, using the XiO TPS (Version 5.0, Elekta Inc., St. Louis, MO). To account for the tissue density change in proton beam path at the presence of the tumor motion, IGTV was overridden to a proton stopping power ratio of tumor tissue, about 1.01 based on sampling of over 10 lung patients treated at our center. In addition, a smearing of $10 \mathrm{~mm}$ was used in compensator calculation for all lung cancer treatment planning. 2-4 fields were employed in a treatment plan. Target coverage and doses to normal tissues such as spinal cord, heart and lung, were evaluated to optimize the beam angle and beam weight for optimal dose distribution. Most patients were treated with 2 CGE (Cobalt Gray equivalent) per fraction for a total dose of from 60 to 74 CGE. A small portion of patients were treated with hypo-fractionation, such as 10 CGE by 3 fractions, 10 CGE by 4 fractions, and 12.5 CGE by 4 fractions.

Treatment delivery. Each patient was treated using uniform scanning proton beams exclusively at our center. The proton therapy unit was made by IBA (Louvain-La-Neuve, Belgium). During the treatment, patients were set up using the same immobilization device as CT simulation and positioned as closely as possible to simulation. Orthogonal $\mathrm{x}$-ray images were used to align and verify the patient positioning and setup every day before treatment. Proton beam was delivered layer by layer with the deepest layer delivered first. The beam delivery time was typically 1 minute or less per beam, and the total patient in room time was about half hour or less for both setup and beam delivery.

\section{Adaptive planning}

Adaptive treatment planning was performed to ensure optimal target coverage and normal tissue sparing when patient anatomy or tumor motion changed during the course of treatment for lung cancer patients. For each patient, repeated 4D CT scanning was performed after patients received about $14 \mathrm{CGE}, 30 \mathrm{CGE}$, and $50 \mathrm{CGE}$. The new CT image set was registered to the initial CT data, using the VelocityAI software system (Version 3.1.0, Varian Medical Systems, Palo Alto, CA). Contours were copied from the initial $\mathrm{CT}$ to the new $\mathrm{CT}$, and modified as needed. A quality assurance (QA) plan was then generated by applying the same proton beams in the original plan to the registered new CT dataset. Physicists and physicians would review the QA plan and determine whether plan adaptation was needed based on several clinical and practical considerations: the PTV coverage and normal tissue dose in the QA plan, the dose deviation from the original plan, the number of fractions remained, and the effort and cost to implement the adaptive plan (e.g., whether new hardware fabrication was involved). If plan change was determined necessary, plan adaptation was applied by either adjust proton ranges for certain beams, adjust beam weight, or replanning with the newly repeated 4D CT. The adapted plan would be used to treat the patient as soon as possible after necessary QA and hardware fabrication. The process of treatment, QA CT, QA planning, and plan adaptation will be repeated until the patient complete the treatment course. A clinical workflow of the adaptive planning for lung cancer treatment at our center is shown in figure 1 .

\section{Patients and toxicities}

In total, we analyzed 165 patients that were treated at our proton therapy center. Among them, $62 \%$ of patients were male and $28 \%$ female. $14 \%$ of patients had small cell lung cancer, and $86 \%$ of patients had non-small cancer. The disease stage ranged from stage IA to stage IV. The patients ranged from 49 to 91 years old, with a mean age of 70.8 years old.

The toxicity data was obtained from the research database that was maintained by the Proton Collaborative Group (PCG). Toxicity analysis was based on the 143 out of 
165 patients who were enrolled in the PCG protocols. The patients undergoing adaptive planning were manually identified and their toxicity data was analyzed separately for comparison with patients without adaptive planning.

\section{Results}

\section{Adaptive planning}

In total 32 adaptive plans (19\%) were made, with one patient experiencing plan adaptation twice. The main reasons leading to plan adaptation include tumor shrinkage (17), tumor growth (6), pleural effusion (3), weight loss (2), and others (3). The plan adaptation strategies include range change only (18), range and modulation change (3), range, modulation, and weight change (2), and replanning (9) (Figure 2).

The time when a plan adaptation was made ranged from the $1^{\text {st }}$ fraction to $31^{\text {st }}$ fraction, with average of about 3 weeks from treatment start, i.e., at about the $17^{\text {th }}$ fraction. The time from QA CT was scanned to the $1^{\text {st }}$ treatment with the adaptive plan ranged from 1 to 2 days when no new hardware was needed, and 3-4 days when it was re-planned with new hardware, i.e., apertures and/or range compensators.

\section{Toxicities}

The toxicity rate for lung cancer patients with and without adaptive planning is shown in figure 3. Dermatitis is the most common toxicity for both patients with and without adaptive planning, followed by esophagitis, hoarseness and constipation for patient with adaptive planning, and fatigue, cough, esophagitis for patients without adaptive planning. The majority of toxicities are Grade 1 and Grade 2. Grade 3 occurrence was very rare Grade 3 and no Grade 4 toxicity was found.
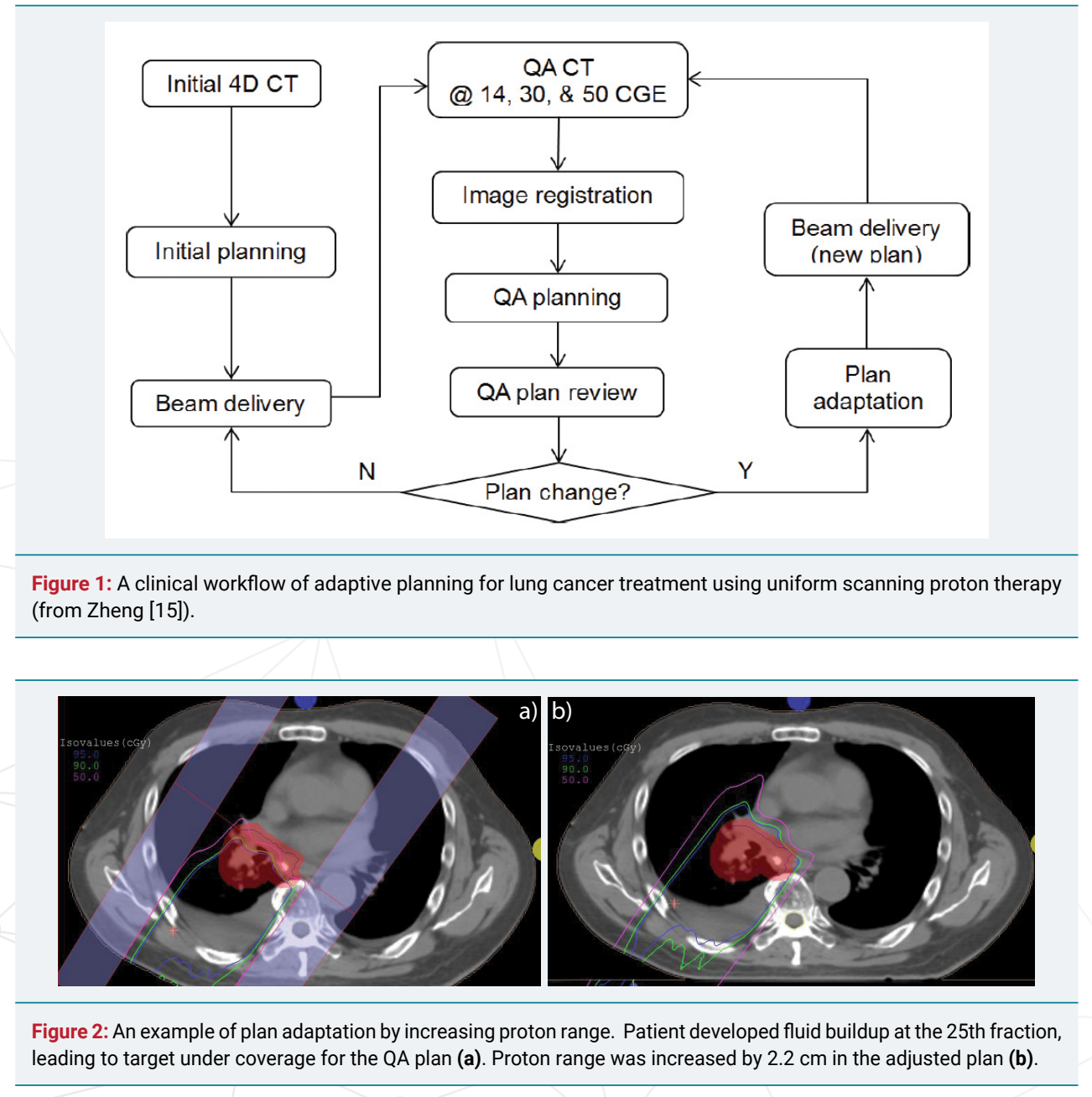


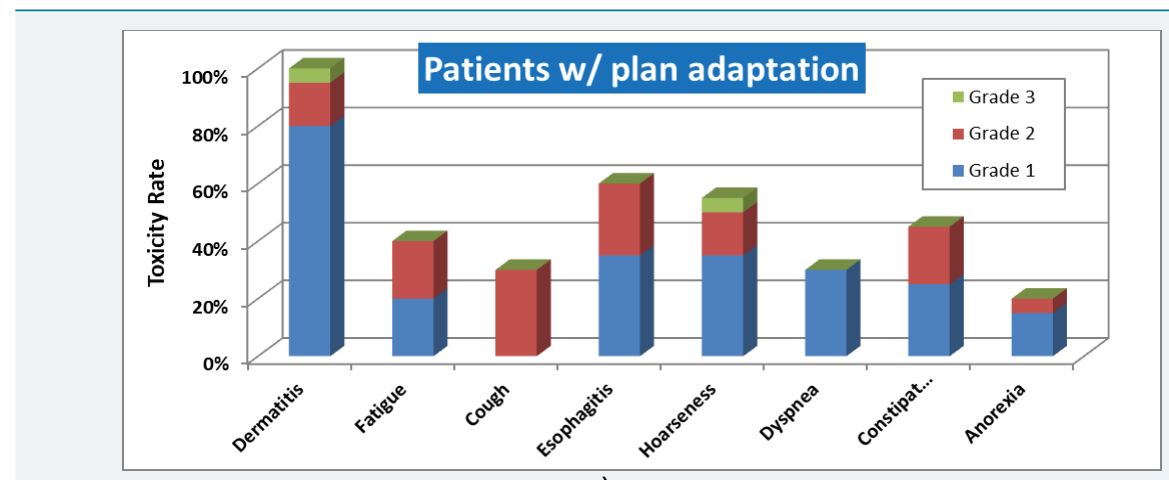

a)

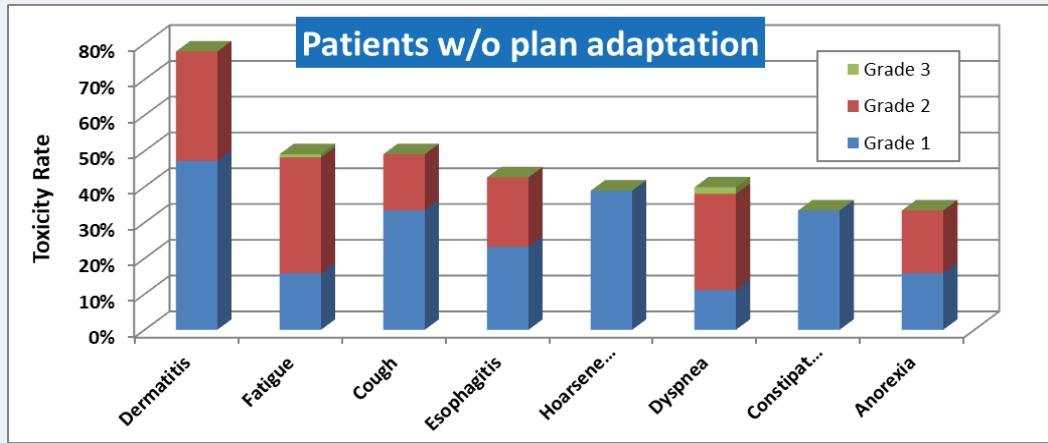

b)

Figure 3: Toxicity rate for lung cancer patients treated with USPT. a) with adaptive planning. b) without adaptive planning.

\section{Discussion}

In this study, we presented our findings on adaptive planning for lung cancer therapy using USPT, including clinical workflow, practical considerations and toxicities. To our knowledge, the study is the first to report such findings on adaptive lung cancer treatment using USPT. About 19\% of patients in this study underwent adaptive planning using USPT, which is similar to what was reported using other proton therapy techniques. For lung cancer treatment with PSPT, Koay et al. reported that $20.5 \%$ of lung cancer patients underwent adaptive planning [16]. For PBS or IMPT, Chang et al. reported that $26.5 \%$ patients were re-planed [17]. Please note that the strategy used for plan adaptation depends largely on the institutional practice and the beam delivery technique used for lung cancer treatment. Re-planning based on the new CT data is a straightforward way commonly used for adaptive treatment for both PSPT and PBS and can also be used for USPT. However, USPT delivers a homogeneous dose to target region, and the proton range can be easily adjusted, so we can adapt the plan by simply adjusting the proton range (and/or modulation width) to maintain similar dose coverage due to anatomy change for certain cases.

The criteria on whether to adapt a plan can vary from institution to institution as well as the individual physicist or physician who reviews the QA plan. At our center, several factors were considered to decide a plan change: 1) whether the target dose coverage and normal tissue dose meet the dose constraints; 2) how much the changes are in target dose coverage and normal tissue dose between the QA plan and initial plan; 3) how many fractions are left; 4) how easily and how quickly to adapt a plan, e.g., whether it is a simple range/modulation change or a replan that needs new hardware fabrication; and 5) what the performance status and treatment goal of the patient and potential clinical impact for the patient are. While plan adaptation would typically improve dosimetric distribution of a treatment plan, it is also associated to certain costs, such as the hardware fabrication, extra efforts to make plan adaptation, 
and potential treatment errors and safety concern associated with any plan change. Therefore, it is our recommendation to consider other clinical and practical factors in addition to DVH endpoints when we decide on whether to make a plan adaptation.

It is critical to fully understand the limitations in plan adaptation process and software tools when evaluating a QA plan. The patient setup in QA CT could be different from the initial CT scan, the image registration software may be not accurate, and the QA plan generation may be incorrect. For example, our treatment plan system does not account for the patient pitch and roll when a QA plan is applied to the new CT data. Also, the beam isocenter for the QA plan was manually placed by a dosimetrist based on image registration, which could be incorrectly placed, especially when different isocenters were used for an initial and boost plan. These limitations or errors in the image registration and QA plan process could result in artificial dose deviation unrelated to anatomy change, leading to unnecessary or incorrect plan adaptation. Therefore, our practice is to always analyze the anatomy change and the correlation between the dose change and anatomy change when we evaluate the QA plan and its dose distribution. If noticeable dose change is in the QA plan but not correlated patient anatomy/motion change or setup variation, it is likely that the dose change is artificial as a result of software limitations or human errors, and further investigation should be warranted.

The top three toxicities for lung cancer therapy using USPT are dermatitis, esophagus, and fatigue, and no Grade 4 toxicity was found in our study. These findings are similar to those for patients treated using PSPT $[16,18]$. Pneumonitis was rare among the patients in the study; however, it was not entered in the PCG database, therefore not included in our toxicity analysis. Overall, we believe USPT is an effective and safe treatment for lung cancer patients.

Currently, offline adaption has been used at our center and most other proton centers, mainly because of lack of in room CT imaging, the long turnaround of manufacturing patient specific device such as apertures and compensators, and lack of better tools on QA plan generation and dose accumulation. However, pencil beam scanning has been a mainstream in newly constructed proton centers, in room cone beam CT or CT on rails are becoming available $[12,15,19]$, and better tools are being developed in image registration, dose accumulation, and plan adaptation. These advancements would make online adaptation for proton therapy possible in the future.

\section{Conclusion}

In conclusion, USPT is a safe treatment for lung cancer patients. Adaptive planning is necessary in proton therapy to account for anatomy change and its effect on proton penetration depth during the course of treatment. It is important to take practical considerations into account and fully understand the limitations of plan adaptation process and tools to make wise decision on adaptive planning.

\section{References}

1. DeLaney TF, Kooy HM. Proton and Charged Particle Radiotherapy. 2015: Wolters Kluwer Health.

2. Paganetti H. Proton Therapy Physics. 2011: Taylor \& Francis. Ref.: https://goo.gl/dSHSA5

3. Zheng $Y$, Ramirez E, Mascia A, Ding $X$, Okoth $B$, et al. Commissioning of output factors for uniform scanning proton beams. Med Phys. 2011; 38: 2299-2306. Ref.: https://goo.gl/5A11nN

4. Cushman TR, Verma V, Rwigema JM. Comparison of proton therapy and intensity modulated photon radiotherapy for locally advanced non-small cell lung cancer: considerations for optimal trial design. J Thorac Dis. 2018; 10(Suppl 9): S988-S990. Ref.: https://goo.gl/hDJ5cw

5. Wu CT, Motegi A, Motegi K, Hotta K, Kohno R, et al. Dosimetric comparison between proton beam therapy and photon radiation therapy for locally advanced non-small cell lung cancer. Jpn $\mathrm{J}$ Clin Oncol. 2016; Ref.: https://goo.gl/zRAoAi 
6. Ohno $\mathrm{T}$, Oshiro $\mathrm{Y}$, Mizumoto $\mathrm{M}$, Numajiri $\mathrm{H}$, Ishikawa $\mathrm{H}$, et al. Comparison of dose-volume histograms between proton beam and X-ray conformal radiotherapy for locally advanced non-small-cell lung cancer. J Radiat Res. 2015; 56: 128-133. Ref.: https://goo.gl/ZAEV5J

7. Stuschke M, Kaiser A, Pöttgen C, Lübcke W, Farr J. Potentials of robust intensity modulated scanning proton plans for locally advanced lung cancer in comparison to intensity modulated photon plans. Radiother Oncol. 2012; 104: 45-51. Ref.: https://goo.gl/vR3bxv

8. Seco J, Panahandeh HR, Westover K, Adams J, Willers H. Treatment of non-small cell lung cancer patients with proton beam-based stereotactic body radiotherapy: dosimetric comparison with photon plans highlights importance of range uncertainty. Int J Radiat Oncol Biol Phys. 2012; 83: 354361. Ref.: https://goo.gl/9qDbxL

9. Hoppe BS, Huh S, Flampouri S, Nichols RC, Oliver KR, et al. Double-scattered proton-based stereotactic body radiotherapy for stage I lung cancer: a dosimetric comparison with photon-based stereotactic body radiotherapy. Radiother Oncol. 2010; 97: 425-430. Ref.: https://goo.gl/fC33ym

10. Kadoya N, Obata Y, Kato T, Kagiya M, Nakamura T, et al. Dose-volume comparison of proton radiotherapy and stereotactic body radiotherapy for non-small-cell lung cancer. Int $\mathrm{J}$ Radiat Oncol Biol Phys. 2011; 79: 1225-1231. Ref.: https://goo.gl/PfccUY

11. Chang JY, Zhang X, Wang X, Kang Y, Riley B. et al. Significant reduction of normal tissue dose by proton radiotherapy compared with three-dimensional conformal or intensity-modulated radiation therapy in Stage I or Stage III non-small-cell lung cancer. Int J Radiat Oncol Biol Phys. 2006; 65: 10871096. Ref.: https://goo.gl/CFxHxe

12. Veiga C, Janssens G, Teng CL, Baudier T, Hotoiu L, et al. First Clinical Investigation of Cone Beam Computed Tomography and Deformable Registration for Adaptive Proton Therapy for Lung Cancer. Int J Radiat Oncol Biol Phys. 2016; 95: 549-559. Ref.: https://goo.gl/EoS9hd

13. Zhang X1, Li Y, Pan X, Xiaoqiang L, Mohan R, et al. Intensity-modulated proton therapy reduces the dose to normal tissue compared with intensity-modulated radiation therapy or passive scattering proton therapy and enables individualized radical radiotherapy for extensive stage IIIB non-smallcell lung cancer: a virtual clinical study. Int J Radiat Oncol Biol Phys. 2010; 77: 357-366. Ref.: https://goo.gl/iDdbVs

14. Zheng Y. Adaptive Radiotherapy for Lung Cancer Using Uniform Scanning Proton Beams, in Radiotherapy, C. Onal, Editor. 2017; IntechOpen. Ref.: https://goo.gl/kbSt3v

15. Zhang M, Zou W, Teo BK. Image guidance in proton therapy for lung cancer. TransI Lung Cancer Res. 2018; 7: 160-170. Ref.: https://goo.gl/vSfn6B

16. Koay EJ, Lege $D$, Mohan R, Komaki R, Cox JD, et al. Adaptive/nonadaptive proton radiation planning and outcomes in a phase II trial for locally advanced non-small cell lung cancer. Int $\mathrm{J}$ Radiat Oncol Biol Phys. 2012; 84: 1093-1000. Ref.: https://goo.gl/bqi4TP

17. Chang JY, Li H, Zhu XR, Liao Z, Zhao L, et al. Clinical implementation of intensity modulated proton therapy for thoracic malignancies. Int J Radiat Oncol Biol Phys. 2014; 90: 809-818. Ref.: https://goo.gl/sQLNjc

18. Chang JY, Komaki R, Wen HY, De Gracia B, Bluett JB, et al. Toxicity and patterns of failure of adaptive/ ablative proton therapy for early-stage, medically inoperable non-small cell lung cancer. Int $\mathrm{J}$ Radiat Oncol Biol Phys. 2011; 80: 1350-1357. Ref.: https://goo.gl/cjm9G4

19. Hua C, Yao W, Kidani T, Tomida K, Ozawa S, et al. A robotic C-arm cone beam CT system for image-guided proton therapy: design and performance. Br J Radiol. 2017. 90: 20170266. Ref.: https://goo.gl/XvBRjZ 